\title{
Novel and highly informative Capsicum SSR markers and their cross-species transferability
}

\author{
G.S.C. Buso ${ }^{1}$, A.M.M. Reis ${ }^{2}$, Z.P.S. Amaral ${ }^{1}$ and M.E. Ferreira ${ }^{1}$ \\ ${ }^{1}$ Laboratório de Genética de Plantas, \\ EMBRAPA Recursos Genéticos e Biotecnologia, Brasília, DF, Brasil \\ ${ }^{2}$ Laboratório de Biotecnologia, \\ Programa de Pós-Graduação em Ciências Genômicas e Biotecnologia, \\ Universidade Católica de Brasília, Brasília, DF, Brasil \\ Corresponding author: G.S.C. Buso \\ E-mail: glaucia.buso@embrapa.br
}

Genet. Mol. Res. 15 (3): gmr.15038689

Received April 1, 2016

Accepted June 9, 2016

Published September 23, 2016

DOI http://dx.doi.org/10.4238/gmr.15038689

Copyright (C) 2016 The Authors. This is an open-access article distributed under the terms of the Creative Commons Attribution ShareAlike (CC BY-SA) 4.0 License

\begin{abstract}
This study was undertaken primarily to develop new simple sequence repeat (SSR) markers for Capsicum. As part of this project aimed at broadening the use of molecular tools in Capsicum breeding, two genomic libraries enriched for $\mathrm{AG} / \mathrm{TC}$ repeat sequences were constructed for Capsicum annuиm. A total of 475 DNA clones were sequenced from both libraries and 144 SSR markers were tested on cultivated and wild species of Capsicum. Forty-five SSR markers were randomly selected to genotype a panel of 48 accessions of the Capsicum germplasm bank. The number of alleles per locus ranged from 2 to 11 , with an average of 6 alleles. The polymorphism information content was on average 0.60 , ranging from 0.20 to 0.83 . The cross-species transferability to seven cultivated and wild Capsicum species was
\end{abstract}


tested with a set of 91 SSR markers. We found that a high proportion of the loci produced amplicons in all species tested. C. frutescens had the highest number of transferable markers, whereas the wild species had the lowest. Our results indicate that the new markers can be readily used in genetic analyses of Capsicum.

Key words: Microsatellites; Pepper; Sweet-pepper; SSR markers; Cross-species transferability; Capsicum annuит

\section{INTRODUCTION}

Capsicum, a genus of the family Solanaceae, contains several species that are grown for food consumption, as well as for medicinal, ornamental or even security purposes. Their fruit, commonly known as pepper, is the most important spice in the world. The top pepper producing areas are South and Central America, India, Hungary, Spain, Italy, and the Southwestern United States (Prince et al., 1993). The cultivated Capsicum species include $C$. annuum, $C$. frutescens, $C$. baccatum, $C$. pubescens, and $C$. chinense. From the agricultural perspective, the most important cultivated species is $C$. annuum, which originated in Mexico (Paran et al., 1998).

Genetic variability within a taxon is of great importance for plant breeding. In Capsicum, variability and relatedness have long been estimated using polymorphism of morphological traits (Prince et al., 1992). More recently, the detection of DNA polymorphisms, as assessed by molecular markers, have become the standard procedure to study genetic variation. Capsicum genetic variation has been studied using different types of molecular markers, including isozyme variants (McLeod et al., 1979), restriction fragment length polymorphisms (Prince et al., 1992), random amplified polymorphic DNA (Paran et al., 1998), amplified fragment length polymorphism (Bradeen and Simon, 1998), inter-simple sequence repeat markers (Dias et al., 2013), as well as microsatellites. Simple sequence repeats (SSRs), or microsatellites, are useful and powerful molecular markers with manifold applications. The potential use of these molecular markers is well documented for Capsicum and they have been extensively used for genetic mapping, assessment of overall genetic variation, population and evolutionary genetic studies, and cultivar identification.

Several reports describe the development of microsatellite markers for use in genetic studies of Capsicum (Sanwen et al., 2000; Lee et al., 2004; Minamiyama et al., 2006; Yi et al., 2006; Nagy et al., 2007; Portis et al., 2007; Kong et al., 2012; Sugita et al., 2013). However, it can be noted that the number of publicly available molecular markers based on polymerase chain reaction (PCR) for peppers, including SSR markers, is still limited. Most of the SSRs included in published Capsicum genetic maps are proprietary markers (e.g., Syngenta, DNA Landmarks, and Seminis - Kong et al., 2012; and VegMarks - http://vegmarks.nivot.affrc. go.jp/, Sugita et al., 2013). Recently, Nicolaï et al. (2012) sequenced the C. annuum cv. 'Yolo Wonder' transcriptome using Roche 454 pyrosequencing. They identified 11,849 single nucleotide polymorphisms and 853 putative SSRs (Nicolaï et al., 2012). However, to date, to our knowledge, there are no reports of genotyping tests using these markers. In addition, Cheng et al. (2016) described a large set of unique microsatellite primer pairs derived from in silico evaluation of DNA sequences of six different pepper genomes (nuclei, mitochondria,

Genetics and Molecular Research 15 (3): gmr.15038689 
and chloroplasts). They tested a random set of 160 primer pairs of which only 65 exhibited polymorphisms among the 21 pepper genotypes analyzed. Hence, there is a need for additional publicly available microsatellite markers that are tested and validated for extensive genotyping application in Capsicum.

It should also be considered that the majority of the marker development studies have restricted the DNA analysis of new markers to C. annuum and some of the cultivated pepper species. Very little is therefore known about application of such markers in experiments with wild relatives of Capsicum. Marker-assisted selection could be used to unveil the potential use of wild species as donors of agronomic important traits to cultivated species, such as disease resistance or abiotic stress tolerance. Cross-species SSR marker transferability tests seem, therefore, to be justifiable in pepper species not only for breeding purposes, but also to stimulate the domestication of species with market potential.

In the present study, we described the development of new microsatellite markers for use in genetic analysis of Capsicum using DNA libraries enriched for AG/TC repeat sequences. The level of polymorphism and the number of alleles at the identified SSR loci were assessed in different accessions from a germplasm collection. In addition, the extent of primer amplification across a broad range of species was examined to determine the potential utility of the SSR markers in other cultivated and wild Capsicum species.

\section{MATERIAL AND METHODS}

\section{Plant material and DNA extraction}

Total genomic DNA was extracted from expanded leaves of a single Capsicum annuum plant (CNPH 4109) and used to construct the genomic DNA libraries. For the SSR marker characterization, 48 accessions of $C$. annuum from different countries (Table 1) were selected from Embrapa's germplasm collection. For the marker cross-species transferability evaluation, two plants from each of $C$. annuum, C. baccatum, C. chinense, C. frutescens, and one plant from each of the wild Capsicum species (C. recurvatum, C. flexuosum, $C$. campylopodium, and Capsicum sp accession LBB1551) were used for amplicon detection. In all experiments, total genomic DNA extraction followed the standard CTAB procedure (Doyle and Doyle, 1987).

\section{SSR-enriched genomic library construction and screening}

Initially, two enriched genomic libraries from a single individual plant of $C$. annuum were constructed with Tsp509 and Mse digested DNA, following the protocols described by Brondani et al. (1998) and Buso et al. (2006). Fragments between 280 and 600 bp were recovered by DEAE-cellulose membrane via electrophoresis. After purification, around 30$\mu \mathrm{g}$ fragments were recovered and ligated to adaptors with Tsp509 and Mse ends. Fragments containing SSR sequences were selected by hybridization with biotinylated oligonucleotides complementary to the repetitive sequence AG/CT, and recovered by magnetic beads linked to streptavidin. The complex DNA-magnetic beads were amplified by PCR through the utilization of primers complementary to the adaptors. After purification, the fragments were cloned in the plasmid vector pGEM-T and then transformed into Escherichia coli strain XL1-Blue.

Genetics and Molecular Research 15 (3): gmr.15038689 
Table 1. Capsicum annuum accessions used to characterize the microsatellite loci.

\begin{tabular}{|c|c|c|}
\hline Accession code & Accession name & Origin \\
\hline 181 & Pimentão Italiano & Local variety - SP - Brazil \\
\hline 184 & PI 123469 & India \\
\hline 187 & PI 201234 & Mexico \\
\hline 191 & Agronômico 10G & Commercial cultivar - Asgrow - Brazil \\
\hline 192 & Magda & Commercial cultivar - Agroflora - Brazil \\
\hline 193 & Yolowonder & Commercial cultivar - Ferry Morse - USA \\
\hline 196 & Cascadura Ykeda & Commercial cultivar - Agroflora - Brazil \\
\hline 199 & Ambato & Argentina \\
\hline 200 & Vyuco & Argentina \\
\hline 291 & Golden Calwonder & USA \\
\hline 295 & Keystone resist. Giant 3 & USA \\
\hline 296 & California Wonder 300 & Commercial cultivar - Asgrow - Brazil \\
\hline 332 & Margareth & Local variety - Brazil \\
\hline 337 & São Carlos & Local variety - Brazil \\
\hline 577 & Early Calwonder & USA \\
\hline 580 & Lito & Netherlands \\
\hline 582 & Latino & Netherlands \\
\hline 641 & Paradicsomalaku & uncertain \\
\hline 642 & Javitott Cecei & uncertain \\
\hline 649 & Plant C-1 & uncertain \\
\hline 678 & PI 140363 & Iran \\
\hline 680 & PI 159266 & USA \\
\hline 689 & PI 169110 & Turkey \\
\hline 693 & PI 169134 & Turkey \\
\hline 703 & PI 183441 & India \\
\hline 707 & PI 194909 & Ethiopia \\
\hline 716 & PI 241646 & Peru \\
\hline 753 & CNPH & Brazil \\
\hline 736 & PI 357587 & Yugoslavia \\
\hline 751 & Amador F1 & Brazil \\
\hline 754 & Salmoai & Senegal \\
\hline 756 & UFRJ-A & Brazil \\
\hline 764 & UFRJ-B & Brazil \\
\hline 823 & Linea 10 & Spain \\
\hline 1379 & XVR 3-25 & USA \\
\hline 1387 & Mallorca & Uncertain \\
\hline 1405 & Yugoslavia Doce & Brazil \\
\hline 1716 & Antibois & France \\
\hline 2171 & NCMA (306) & USA \\
\hline 2275 & Cascadura Ikeda & Commercial cultivar - Agroceres - Brazil \\
\hline 2282 & PM3 F1 & Commercial cultivar - Agroceres - Brazil \\
\hline 2474 & Bel Rubi & Brazil \\
\hline 2519 & Marconi & France \\
\hline 2678 & Chili Ancho & USA \\
\hline 2691 & Pimiento & USA \\
\hline 2732 & Hibrido Fortuna & Brazil \\
\hline 2764 & Hot Poken F2 & Germany \\
\hline Pimentão 3 & $\mathrm{CNPH}$ & Brazil \\
\hline
\end{tabular}

Hybridization screening for microsatellites, sequencing of positive clones, and primer design

Aliquots from the enriched library were plated for screening on agar plates 
containing ampicillin and tetracycline. Recombinant colonies with SSR were identified by hybridization with oligonucleotide probes poly dAdG/dCdT labeled with digoxigenin11-ddUTP using a DIG oligonucleotide 3'-end labeling kit (Boehringer Mannheim, Indianapolis, IN, USA) following the manufacturer instructions. After high stringency washing, the membranes were processed for chemiluminescent detection and exposed to $\mathrm{X}$-ray film for $2-3 \mathrm{~h}$ at $37^{\circ} \mathrm{C}$.

Positive clones were grown overnight in liquid LB-ampicillin medium. Plasmid DNA was extracted by alkaline lysis (Sambrook et al., 1989), PCR-amplified, and purified, after which inserts were sequenced on an Applied Biosystems 3700 (Perkin-Elmer, San Jose, CA, USA) DNA sequencer. Primer pairs complementary to sequences flanking the repeat element were designed using the Primer 3 software (Untergasser et al., 2012) set to the following stringency criteria: a primer annealing temperature (Ta) range from $52^{\circ}$ to $58^{\circ} \mathrm{C}$; a primer pair Ta variance of less than $3^{\circ} \mathrm{C}$; $\mathrm{GC}$ content ranging from 40 to $60 \%$; and absence of complementarity between primers.

\section{Primer screening and PCR conditions}

Microsatellite amplification was performed in a $13-\mu \mathrm{L}$ reaction mix containing 0.3 $\mu \mathrm{M}$ each primer, $1 \mathrm{U}$ Taq DNA polymerase, $0.2 \mathrm{mM}$ each dNTP, $10 \mathrm{mM}$ Tris- $\mathrm{HCl}, 50 \mathrm{mM}$ $\mathrm{KCl}, 1.5 \mathrm{mM} \mathrm{MgCl}{ }_{2}, \mathrm{DMSO} 50 \%$, and $7.5 \mathrm{ng}$ template DNA. Amplification was performed with the cycling conditions: $96^{\circ} \mathrm{C}$ for $2 \mathrm{~min} ; 29$ cycles of $94^{\circ} \mathrm{C}$ for $1 \mathrm{~min}, 54^{\circ}-72^{\circ} \mathrm{C}$ (based on each primer pair Ta) for $1 \mathrm{~min}, 72^{\circ} \mathrm{C}$ for $1 \mathrm{~min}$; and $72^{\circ} \mathrm{C}$ for $7 \mathrm{~min}$. The Ta for each primer pair was optimized to produce clear and robust DNA band amplification. Each primer pair was screened for product polymorphism on 3\% agarose gels stained with ethidium bromide and size compared to a 1-kb and 100-bp DNA ladder.

\section{Transferability analysis}

To test the cross-species transferability of the SSR loci, we used two plants of each of the cultivated species and one plant of each of the Capsicum wild species. There were no additional attempts to optimize PCR conditions in cross-species transferability tests.

\section{SSR locus characterization}

The primer pairs showing high-quality PCR-amplified products at the given annealing temperature were used for estimates of allele sizes and for genotype determination. PCR products were separated on $4 \%$ denaturing polyacrylamide gels stained with silver nitrate (Bassam et al., 1991) and sized by comparison to a 10-bp DNA ladder. Forty-five primer pairs were used on a set of 48 accessions of the Capsicum collection. The number of alleles and allelic diversity were surveyed at each locus. To evaluate the informativeness of markers, the polymorphic information content (PIC), a statistic that incorporates both the number of alleles and the frequency of detection of alleles among the genotypes utilized, was calculated following Botstein et al. (1980).

Genetics and Molecular Research 15 (3): gmr.15038689 


\section{RESULTS}

\section{SSR development}

A total of 1000 recombinant colonies of $C$. annuum-enriched genomic library were screened with an AG/TC probe and 475 clones were detected that were positive for microsatellite-containing regions. The DNA sequencing indicated that $259(55 \%)$ clones contained useful sequences for primer design, showing an adequate number of high-quality nucleotides flanking the repeat sequence. Three different categories of repeats were evident in the sequenced clones (Table 1): a) 217 perfect repeats, with no interruptions, ranging from 9 to 44 dinucleotide repeat units; b) 24 compound repeats, where two or more repeats were adjacent to each other; c) 18 imperfect repeats, with interruption in the repeat sequence. Since the library was enriched for (AG) repeats, this was by far the most common repeated motif present in almost all the microsatellite sequences. Nevertheless, (CA) tandems were also observed associated to (GA) repeats in compound sequences.

\section{SSR locus characterization}

A total of 144 SSR markers were tested with the DNA used to develop the two DNA genomic libraries. As many as 118 markers amplified very clean and easily interpretable PCR products and did not require further adjustment of the PCR conditions. However, 26 markers showed some non-specific amplification of secondary bands, did not amplify any product, or resulted in bands that could not be interpreted. For this last group of markers, further optimization of the reaction conditions was attempted that will be reported elsewhere.

The PIC for a selected sample of 45 SSR markers was estimated using a set of 48 genotypes of $C$. annuum. Among the 45 loci tested, the number of alleles per locus ranged from 2 to 11, with an average of 6 alleles per locus (Table 2). The PIC value was on average 0.60 , ranging from 0.20 to 0.83 .

The number of perfect repeats correlated weakly with the PIC values $\left(\mathrm{r}^{2}=0.15\right)$. For instance, primer CaBR64 had 42 repeats and a PIC of 0.81, primer CaBR61 had 29 repeats and the highest PIC value of 0.83 , whereas CaBR 40 had 23 repeats and a PIC of 0.81 .

\section{Transferability}

Ninety-one SSR markers were tested on four cultivated and four wild Capsicum species (Table 3 ). The cultivated species with the highest number of markers showing amplicon products was $C$. frutescens (85), followed by $C$. chinense (84) and $C$. baccatum (79). Two markers amplified PCR products only in $C$. annuum. The wild species with the highest number of markers showing amplicon products were C. recurvatum and Capsicum sp LBB1551, both with 63 markers, followed by C. campylopodium (55 markers) and C. flexuosum (45 markers). PCR products were obtained for 73 SSR markers (80\%) in all cultivated species and 41 markers in all wild species. Twenty-three markers did not show PCR products in any of the wild Capsicum species tested.

Genetics and Molecular Research 15 (3): gmr.15038689 
Table 2. Primer sequences, repeat motif, fragment size, annealing temperature (Ta), total number of alleles $\left(N_{\mathrm{A}}\right)$, and polymorphic information content (PIC) for a new set of Capsicum annuum SSR markers.

\begin{tabular}{|c|c|c|c|c|c|c|}
\hline Marker & Repeat motif & Primer pair sequences (5'-3') & Fragment size (bp) & $\mathrm{Ta}\left({ }^{\circ} \mathrm{C}\right)$ & $N_{\mathrm{A}}$ & PIC \\
\hline $\mathrm{CaBR} 20$ & $(\mathrm{AG})_{27}$ & $\begin{array}{l}\text { CCgTAAAgAAATCAAACCAC } \\
\text { gCATgCACACATAAACACTC }\end{array}$ & $108-122$ & 56 & 4 & 0.40 \\
\hline CaBR23 & $(\mathrm{TC})_{31}$ & $\begin{array}{l}\text { ggCTCCTAggTATgCACCAg } \\
\text { AATgTgATgCACAgTgCACC }\end{array}$ & $162-174$ & 56 & 7 & 0.77 \\
\hline CaBR24 & $(\mathrm{TC})_{11}$ & $\begin{array}{l}\text { gTTATCTCCTTTTCCCAATC } \\
\text { AAATgTTAggAACTCACCAg }\end{array}$ & $80-90$ & 56 & 2 & 0.30 \\
\hline CaBR25 & $(\mathrm{TC})_{17}$ & $\begin{array}{l}\text { gCCTCTCTCTTATATTTCAg } \\
\text { gTTTAATAggATgACAgAgC }\end{array}$ & $117-129$ & 56 & 5 & 0.57 \\
\hline CaBR30 & $(\mathrm{CT})_{28}$ & $\begin{array}{l}\text { CCCTTTgAAACCTgATCTTg } \\
\text { gATggACTTgATgAgATTgC }\end{array}$ & $108-144$ & 56 & 4 & 0.56 \\
\hline CaBR36 & $(\mathrm{CT})_{22}$ & $\begin{array}{l}\text { gggTAAAggTACTTAgTAgA } \\
\text { gTTgTATTgCTTTAgCTCAg }\end{array}$ & $112-136$ & 56 & 6 & 0.57 \\
\hline CaBR38 & $(\mathrm{CT})_{12}$ & $\begin{array}{l}\text { ATCCggCggAgCTTCATAAC } \\
\text { gCCgATCgATATATggATgC }\end{array}$ & $94-98$ & 56 & 3 & 0.45 \\
\hline CaBR39 & $(\mathrm{CT})_{13}$ & $\begin{array}{l}\text { CATCCATATATCgATCggCT } \\
\text { TTTCgACCATgTTCAgATCC }\end{array}$ & $89-95$ & 56 & 4 & 0.43 \\
\hline CaBR40 & $(\mathrm{CT})_{23}$ & $\begin{array}{l}\text { TCAgACACCAAgCCATCAA } \\
\text { gCAAgCTAATggCATggTA }\end{array}$ & $124-142$ & 56 & 9 & 0.81 \\
\hline CaBR49 & $(\mathrm{AG})_{21}$ & $\begin{array}{l}\text { CTATCTTCgCATATAggCAg } \\
\text { AATCTCTgTggCTgACTCAA }\end{array}$ & $146-154$ & 56 & 4 & 0.37 \\
\hline CaBR53 & $(\mathrm{TC})_{25}$ & $\begin{array}{l}\text { CgACCTTCAggACAgATCAT } \\
\text { CTggTAACCTAgAAAggCAg }\end{array}$ & $220-260$ & 56 & 5 & 0.65 \\
\hline CaBR58 & $(\mathrm{TC})_{14}$ & $\begin{array}{l}\text { CggAgAAgAACTAgACgATT } \\
\text { CTTgACAAACATCCACCACT }\end{array}$ & $153-157$ & 56 & 3 & 0.45 \\
\hline CaBR59 & $(\mathrm{TC})_{17}$ & $\begin{array}{l}\text { CCAgggTACTTTgTCTTTCC } \\
\text { ggAgCCTCggCACATAAA }\end{array}$ & 116-128 & 56 & 3 & 0.37 \\
\hline CaBR61 & $(\mathrm{AG})_{29}$ & $\begin{array}{l}\text { gAAgAgTCCTACTCAATCTA } \\
\text { CTATATggTTTCACTCCTTC }\end{array}$ & $84-134$ & 56 & 11 & 0.83 \\
\hline CaBR63 & $(\mathrm{CT})_{21}$ & $\begin{array}{l}\text { gAgTgCCTATCgATgTCTTT } \\
\text { AgCTATCTAATTgCACCAAg }\end{array}$ & $146-172$ & 56 & 9 & 0.60 \\
\hline CaBR64 & $(\mathrm{AG})_{42}$ & $\begin{array}{l}\text { AgAgTgTCCCATgCATAC } \\
\text { gCTTATTCCAAAggTCTC }\end{array}$ & $114-156$ & 56 & 9 & 0.81 \\
\hline CaBR65 & $(\mathrm{TC})_{24}$ & $\begin{array}{l}\text { gAgggTTTgTCATCTTATTg } \\
\text { CTgAgACAgAAATTCTTgCT }\end{array}$ & $100-136$ & 56 & 4 & 0.64 \\
\hline CaBR67 & $(\mathrm{GA})_{25}$ & $\begin{array}{l}\text { CCgAgAAAATgCACACAA } \\
\text { TgACATACTCTTCCTACAgCTA }\end{array}$ & $90-114$ & 56 & 6 & 0.62 \\
\hline CaBR68 & $(\mathrm{TC})_{21}$ & $\begin{array}{l}\text { TAATAgAAgCCCgCCCCT } \\
\text { ATgCATgAgACTgTTgTTATggTA }\end{array}$ & 114-132 & 56 & 5 & 0.65 \\
\hline CaBR73 & $(\mathrm{AG})_{25}$ & $\begin{array}{l}\text { gCAAggAgAgAATCAAggTT } \\
\text { ggAggTCAACACTTggATTAg }\end{array}$ & $318-320$ & 56 & 2 & 0.50 \\
\hline CaBR75 & $(\mathrm{TC})_{26}$ & $\begin{array}{l}\text { CAACACTAAgTggTCATTCg } \\
\text { CTgAgACAgAAATTCTTgCT }\end{array}$ & $133-171$ & 56 & 6 & 0.65 \\
\hline CaBR76 & $(\mathrm{AG})_{24}$ & $\begin{array}{l}\text { gCTAgTCACgTCAATCTgTT } \\
\text { CATTCTCTTCTTCTCAAACg }\end{array}$ & $116-140$ & 56 & 7 & 0.73 \\
\hline CaBR77 & $(\mathrm{AG})_{24}$ & $\begin{array}{l}\text { gCATggTACTTCTTAgCATT } \\
\text { gACACCAAgCCATCAATTAT }\end{array}$ & $108-122$ & 56 & 6 & 0.74 \\
\hline CaBR79 & $(\mathrm{TC})_{26}$ & $\begin{array}{l}\text { CACTgggTATgTTgTTgTAA } \\
\text { CCgTAAAgAAATCATACCAC }\end{array}$ & $120-138$ & 56 & 4 & 0.45 \\
\hline CaBR80 & $(\mathrm{TC})_{20}(\mathrm{TG})_{7}$ & $\begin{array}{l}\text { CTCAAgTgTgCCAggTgATT } \\
\text { gAgAgACAggAAgAgACgTACA }\end{array}$ & $106-132$ & 56 & 4 & 0.63 \\
\hline CaBR81 & $\begin{array}{l}(\mathrm{TC})_{13}(\mathrm{CA})_{4} \\
(\mathrm{CG})_{6}(\mathrm{TC})_{11} \\
(\mathrm{CA})_{5}(\mathrm{CG})_{5}\end{array}$ & $\begin{array}{l}\text { gCAACTTCTCCgAgACAATC } \\
\text { TTTCTAgTgCATgggACgTA }\end{array}$ & $178-182$ & 56 & 3 & 0.52 \\
\hline CaBR82 & $(\mathrm{AG})_{37}$ & $\begin{array}{l}\text { gCACATgCACgTACAACC } \\
\text { ggggTAAAAggCATTgTg }\end{array}$ & $172-190$ & 56 & 8 & 0.78 \\
\hline CaBR85 & $(\mathrm{TC})_{25}$ & $\begin{array}{l}\text { CTTCACTTTgCTCACCCTAC } \\
\text { TTACTggTTCAAgAgggAAA }\end{array}$ & $116-154$ & 56 & 10 & 0.72 \\
\hline CaBR88 & $(\mathrm{AG})_{22}$ & $\begin{array}{l}\text { AATggATgTTCCCTTgCTTT } \\
\text { CAACTgATCAACCATTCCgT }\end{array}$ & $148-164$ & 56 & 7 & 0.59 \\
\hline CaBR90 & $(\mathrm{AG})_{17}$ & $\begin{array}{l}\text { AAggAggAACAAgAACAACC } \\
\text { ATTggCAAgCACATgTAACT }\end{array}$ & $120-132$ & 56 & 4 & 0.32 \\
\hline CaBR93 & $(\mathrm{AG})_{25}$ & $\begin{array}{l}\text { gCAATATgTTgAgTAgCTgT } \\
\text { ACCCATACAAATCATCCAC }\end{array}$ & $160-182$ & 56 & 6 & 0.79 \\
\hline CaBR97 & $(\mathrm{AG})_{38}$ & $\begin{array}{l}\text { gTgTgTCTgTgTgCATgAgC } \\
\text { gCCTTCAgCTgTggTTATg }\end{array}$ & $130-170$ & 56 & 6 & 0.77 \\
\hline
\end{tabular}

Continued on next page

Genetics and Molecular Research 15 (3): gmr.15038689 
Table 2. Continued.

\begin{tabular}{|c|c|c|c|c|c|c|}
\hline Marker & Repeat motif & Primer pair sequences $\left(5^{\prime}-3^{\prime}\right)$ & Fragment size (bp) & $\mathrm{Ta}\left({ }^{\circ} \mathrm{C}\right)$ & $N_{\mathrm{A}}$ & PIC \\
\hline CaBR98 & $(\mathrm{AG})_{20}$ & $\begin{array}{l}\text { CgAgCTAATggCATggTACT } \\
\text { TCAgACACCAAgCCATCAAT }\end{array}$ & $132-146$ & 56 & 7 & 0.76 \\
\hline CaBR100 & $(\mathrm{AG})_{17}$ & $\begin{array}{l}\text { ATggCATggTACTTCTTAgCA } \\
\text { CCAAgCCATCAATTATgCAA }\end{array}$ & $138-152$ & 56 & 7 & 0.71 \\
\hline CaBR106 & $(\mathrm{AG})_{16}$ & $\begin{array}{l}\text { CgAggAAgCATTTAATgAgA } \\
\text { CCTAgTCAATTggATAgTggg }\end{array}$ & $168-182$ & 56 & 6 & 0.78 \\
\hline CaBR107 & $(\mathrm{AG})_{13}$ & $\begin{array}{l}\text { AAgCTAATggCATggTACTTCT } \\
\text { CCAAgCCATCAATTATgCAA }\end{array}$ & $144-154$ & 56 & 6 & 0.73 \\
\hline CaBR112 & $\begin{array}{l}\text { (TC) }{ }_{19} \mathrm{TA} \\
(\mathrm{TG})_{12}\end{array}$ & $\begin{array}{l}\text { CCTCCATTCTTCTATTgTACAgg } \\
\text { TTgCACCCgCAgATTAgTT }\end{array}$ & 136-142 & 56 & 4 & 0.69 \\
\hline CaBR113 & $(\mathrm{GT})_{13}(\mathrm{GA})_{26}$ & $\begin{array}{l}\text { CAgAACTTAgCAgAggACCAg } \\
\text { ATgTTCTgAgTCCACgATgC }\end{array}$ & $138-190$ & 56 & 5 & 0.48 \\
\hline CaBR115 & $(\mathrm{AG})_{20}$ & $\begin{array}{l}\text { CATgAgACTgTTgTTATgg } \\
\text { TgTCCAgTTTATgTTAgTTA }\end{array}$ & $142-160$ & 56 & 5 & 0.65 \\
\hline CaBR116 & $(\mathrm{AG})_{22}$ & $\begin{array}{l}\text { gCATggTACTTCTTAgCATT } \\
\text { gACACCAAgCCATCAATTA }\end{array}$ & $118-130$ & 56 & 7 & 0.74 \\
\hline CaBR124 & $(\mathrm{TC})_{23}$ & $\begin{array}{l}\text { CgACCTTCAggACAgATCAT } \\
\text { ggCAgAACATACTgAgACAgAA }\end{array}$ & 156-192 & 56 & 5 & 0.63 \\
\hline CaBR136 & $(\mathrm{AG})_{16}$ & $\begin{array}{l}\text { GTggACTAACAgACTCAACg } \\
\text { CTgATgATgCAgAACATgAT }\end{array}$ & $160-166$ & 56 & 3 & 0.64 \\
\hline CaBR137 & $(\mathrm{TC})_{18}$ & $\begin{array}{l}\text { CgTCCTTTATTTCTTgCTT } \\
\text { AAAgAgAgAgATAgAAAggg }\end{array}$ & $64-82$ & 56 & 6 & 0.69 \\
\hline CaBR138 & $(\mathrm{TC})_{27}$ & $\begin{array}{l}\text { ATCCCATTCTCATTTCTAgg } \\
\text { gCAAgCAgTTTTTAgTgAgA }\end{array}$ & 156-158 & 56 & 2 & 0.20 \\
\hline CaBR144 & $(\mathrm{GT})_{5}(\mathrm{GA})_{21}$ & $\begin{array}{l}\text { TAgAAACACAAAATgTCCCC } \\
\text { CCATTgACAAggACAATTCT }\end{array}$ & $116-132$ & 56 & 6 & 0.75 \\
\hline
\end{tabular}

Table 3. Marker denomination, repeat motif, annealing temperature (Ta), primer sequence, and cross-species amplification for 91 microsatellite loci tested with DNA from four cultivated Capsicum species (C. annuиm, C. baccatum, C. chinense, and C. frutescens) and four wild Capsicum species (Capsicum sp accession LBB1551, C. recurvatum, C. flexuosum, and C. campylopodium).

\begin{tabular}{|c|c|c|c|c|c|c|c|c|c|c|c|}
\hline $\begin{array}{l}\text { Marker } \\
\text { CaBR2 }\end{array}$ & $\begin{array}{c}\text { Repeat motif } \\
\text { (CT) } \mathrm{T}_{6}\end{array}$ & $\begin{array}{l}\frac{\mathrm{Ta}\left({ }^{\circ} \mathrm{C}\right)}{60} \\
6\end{array}$ & \begin{tabular}{|l|} 
Primer sequence \\
ACTACCgCCATCTCCAC \\
ATTCgAgTgTTgAAATTAC
\end{tabular} & \begin{tabular}{|c|c|} 
C. anmulum \\
$\mathrm{X}$
\end{tabular} & \begin{tabular}{|c|} 
C. baccatum \\
$\mathrm{X}$
\end{tabular} & $\begin{array}{c}\text { C. chinense } \\
\mathrm{X}\end{array}$ & $\begin{array}{c}\text { C. frutescens } \\
\mathrm{X}\end{array}$ & $\frac{\text { LBB1551 }}{\mathrm{X}}$ & \begin{tabular}{|c|} 
C. recurvatum \\
$\mathrm{X}$
\end{tabular} & \begin{tabular}{|c} 
C. flexuosum \\
$\mathrm{X}$
\end{tabular} & $\frac{\text { C. campylopodium }}{\mathrm{x}}$ \\
\hline CaBR3 & (TC)18 & 54 & \begin{tabular}{|l} 
CTAgTTACCTTACTTTCTCTCTC \\
AgCCACgACAATCAATAg \\
\end{tabular} & $\mathrm{x}$ & $\mathrm{x}$ & $\mathrm{x}$ & $\mathrm{x}$ & $\mathrm{x}$ & $\mathrm{x}$ & $\mathrm{x}$ & $\mathrm{x}$ \\
\hline CaBR4 & $(\mathrm{AG})_{14}$ & 56 & \begin{tabular}{|l|} 
CATgATgTTAagAgAgAAgA \\
ATCTCATCTCCTTTCTCTTT
\end{tabular} & $\mathrm{x}$ & $\mathrm{x}$ & $\mathrm{x}$ & $\mathrm{x}$ & $\mathrm{x}$ & $\mathrm{x}$ & $\mathrm{x}$ & $\mathrm{x}$ \\
\hline CaBR5 & $\left(\mathrm{AT}_{\mathrm{B}} \mathrm{T}_{\mathrm{s}}\right.$ & 54 & \begin{tabular}{|l|l|} 
TgTgTTgACTggTATgTTCC \\
\end{tabular} & $\mathrm{x}$ & $\mathrm{x}$ & $\mathrm{x}$ & $\mathrm{x}$ & $\mathrm{x}$ & $\mathrm{x}$ & $\mathrm{x}$ & $\mathrm{x}$ \\
\hline CaBR10 & $(\mathrm{AG})_{27}$ & 54 & $\begin{array}{l}\text { AAACACgAgEAgAgAgA } \\
\text { CgAgTgTgIgAATATgA }\end{array}$ & $\mathrm{x}$ & - & $\mathrm{x}$ & $\mathrm{x}$ & $\mathrm{x}$ & $\mathrm{x}$ & & $\mathrm{x}$ \\
\hline CaBRII & $(\mathrm{CT})_{12}$ & 56 & gTT gTTATCTCTTTTCCCA & $\mathrm{x}$ & $\mathrm{x}$ & $\mathrm{x}$ & $\mathrm{x}$ & $\mathrm{x}$ & $\mathrm{x}$ & $\mathrm{x}$ & $\mathrm{x}$ \\
\hline CaBR13 & $(\mathrm{CT})_{12}$ & 60 & \begin{tabular}{|l} 
CAgACAATECTCCTTCTC \\
CAATCTTCCCAACTgCTC
\end{tabular} & $\mathrm{x}$ & $\mathrm{x}$ & $\mathrm{x}$ & $\mathrm{x}$ & $\mathrm{x}$ & $\mathrm{x}$ & $\mathrm{x}$ & $\mathrm{x}$ \\
\hline CaBR15 & $(\mathrm{CT})_{10}$ & 50 & $\begin{array}{l}\text { gCTCTTATCAATgAACCA } \\
\text { AAgAAgAggAagAGC }\end{array}$ & $\mathrm{x}$ & $\mathrm{x}$ & $\mathrm{x}$ & $\mathrm{x}$ & & & & $\mathrm{x}$ \\
\hline CaBR17 & (CT)11 & 52 & $\begin{array}{l}\text { CTCATTTCAAATCTCAAg } \\
\text { CTgAATTCCAATTATgTg }\end{array}$ & $\mathrm{x}$ & $\mathrm{x}$ & $\mathrm{x}$ & $\mathrm{x}$ & & & & \\
\hline CaBR20 & $(\mathrm{AG})_{27}$ & 56 & $\begin{array}{l}\text { CCGAAAAgAAATCAACCAC } \\
\text { gCATgCACACATAAACACTC }\end{array}$ & $\mathrm{x}$ & $\mathrm{x}$ & $\mathrm{x}$ & $\mathrm{x}$ & $\mathrm{x}$ & $\mathrm{x}$ & & \\
\hline CaBR22 & $(\mathrm{TC})_{7}$ & 56 & $\begin{array}{l}\text { ATCCTgTTETCTEATCTA } \\
\text { CCAACAgATTgAACTgATA } \\
\end{array}$ & $\mathrm{x}$ & $\mathrm{x}$ & $\mathrm{x}$ & $\mathrm{x}$ & $\mathrm{x}$ & $\mathrm{x}$ & $\mathrm{x}$ & \\
\hline CaBR23 & $(\mathrm{TC})_{31}$ & 56 & $\begin{array}{l}\text { ggCTCCTAggTATgCACCAg } \\
\text { AATgTgATgCACATTCACC }\end{array}$ & $\mathrm{x}$ & $\mathrm{x}$ & $\mathrm{x}$ & $\mathrm{x}$ & $\mathrm{x}$ & $\mathrm{x}$ & $\mathrm{x}$ & $\mathrm{x}$ \\
\hline CaBR24 & (TC) & 56 & $\begin{array}{l}\text { gTTATCTCCTTTTCCCAATC } \\
\text { AAATgTTAggAACTCACCAg }\end{array}$ & $\mathrm{x}$ & $\mathrm{x}$ & $\mathrm{x}$ & $\mathrm{x}$ & $\mathrm{x}$ & $\mathrm{x}$ & & $\mathrm{x}$ \\
\hline CaBR25 & $(\mathrm{TC}) 17$ & 56 & 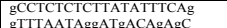 & $\mathrm{x}$ & $\mathrm{x}$ & $\mathrm{x}$ & $\mathrm{x}$ & & & & $\mathrm{x}$ \\
\hline CaBR27 & $\begin{array}{c}(\mathrm{CA})_{122(\mathrm{CT}))_{17 \mathrm{AT}}} \\
\mathrm{CG}(\mathrm{CT}) 9\end{array}$ & 60 & \begin{tabular}{|l} 
gCAgAggaCCAgTTAgCATA \\
TgTTCTgAgTCCACgATgCT
\end{tabular} & $\mathrm{x}$ & $\mathrm{x}$ & $\mathrm{x}$ & $\mathrm{x}$ & & & & \\
\hline CaBR28 & $(\mathrm{CT}) 1(\mathrm{TA})_{6}$ & 56 & \begin{tabular}{|l|} 
ATgATgACCTTgTTgAgCAg \\
TTgTATgTgTgTgTgCgTgT
\end{tabular} & $\mathrm{x}$ & $\mathrm{x}$ & $\mathrm{x}$ & & & & & \\
\hline CaBR30 & $\left(\mathrm{CT}_{28}\right.$ & 56 & $\begin{array}{l}\text { CCCTTIGAAACCTgATCTTg } \\
\text { gATggACTIgATgAgATTEC }\end{array}$ & $\mathrm{x}$ & & & $\mathrm{x}$ & $\mathrm{x}$ & $\mathrm{x}$ & & \\
\hline CaBR31 & (CT) 20 & 56 & $\begin{array}{l}\text { CAACCCTTACAAATCATCCA } \\
\text { TTATTgAgAggagAgaCA }\end{array}$ & $\mathrm{x}$ & $\mathrm{x}$ & $\mathrm{x}$ & $\mathrm{x}$ & $\mathrm{x}$ & $\mathrm{x}$ & $\mathrm{x}$ & $\mathrm{x}$ \\
\hline CaBR34 & $\left(\mathrm{GA}_{21}\right.$ & 54 & \begin{tabular}{|l|l|} 
gggTTAAgCATATgTTgAg \\
\end{tabular} & $\mathrm{x}$ & $\mathrm{x}$ & $\mathrm{x}$ & $\mathrm{x}$ & $\mathrm{x}$ & $\mathrm{x}$ & & \\
\hline CaBR35 & $(\mathrm{CT})_{22}$ & 50 & $\begin{array}{l}\text { TTGGCCAgTTTAAgTTAg } \\
\text { TggTAATATTgTTCTT }\end{array}$ & $\mathrm{x}$ & $\mathrm{x}$ & $\mathrm{x}$ & $\mathrm{x}$ & & & & \\
\hline CaBR36 & $\left(\mathrm{TC}_{22}\right.$ & 56 & 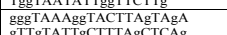 & $\mathrm{x}$ & $\mathrm{x}$ & $\mathrm{x}$ & $\mathrm{x}$ & $\mathrm{x}$ & $\mathrm{x}$ & $\mathrm{x}$ & $\mathrm{x}$ \\
\hline CaBR37 & $(\mathrm{CT})_{16}$ & 56 & 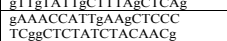 & $\mathrm{x}$ & $\mathrm{x}$ & & $\mathrm{x}$ & & & & \\
\hline CaBR38 & $(\mathrm{CT}) 12$ & 56 & \begin{tabular}{|l|l|l|} 
ATCCggCggAgCTTCATAAC \\
\end{tabular} & $\mathrm{x}$ & $\mathrm{x}$ & $\mathrm{x}$ & $\mathrm{x}$ & $\mathrm{x}$ & & & $\mathrm{x}$ \\
\hline CaBR39 & (CT) 13 & 56 & \begin{tabular}{|l|} 
CATCCATATATCgATCgCT \\
TTTEACCATgTTCAgATCC
\end{tabular} & $\mathrm{x}$ & $\mathrm{x}$ & $\mathrm{x}$ & $\mathrm{x}$ & $\mathrm{x}$ & $\mathrm{x}$ & $\mathrm{x}$ & $\mathrm{x}$ \\
\hline CaBR43 & $\left(\mathrm{TC}_{14}\right.$ & 58 & $\begin{array}{l}\text { AgCCTAACACCATAACCAACCA } \\
\text { ggTgCATgACATTTRAgTTA }\end{array}$ & $\mathrm{x}$ & $\mathrm{x}$ & $\mathrm{x}$ & $\mathrm{x}$ & & & & \\
\hline 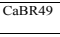 & $(\mathrm{GA})_{21}$ & 56 & \begin{tabular}{|l|} 
CTATCTTCgCATATAggCAg \\
AATCTCTgTggCTgACTCAA
\end{tabular} & $\mathrm{x}$ & $\mathrm{x}$ & $\mathrm{x}$ & $\mathrm{x}$ & & & & \\
\hline
\end{tabular}

Continued on next page

Genetics and Molecular Research 15 (3): gmr.15038689 
Novel informative Capsicum SSRs and their transferability

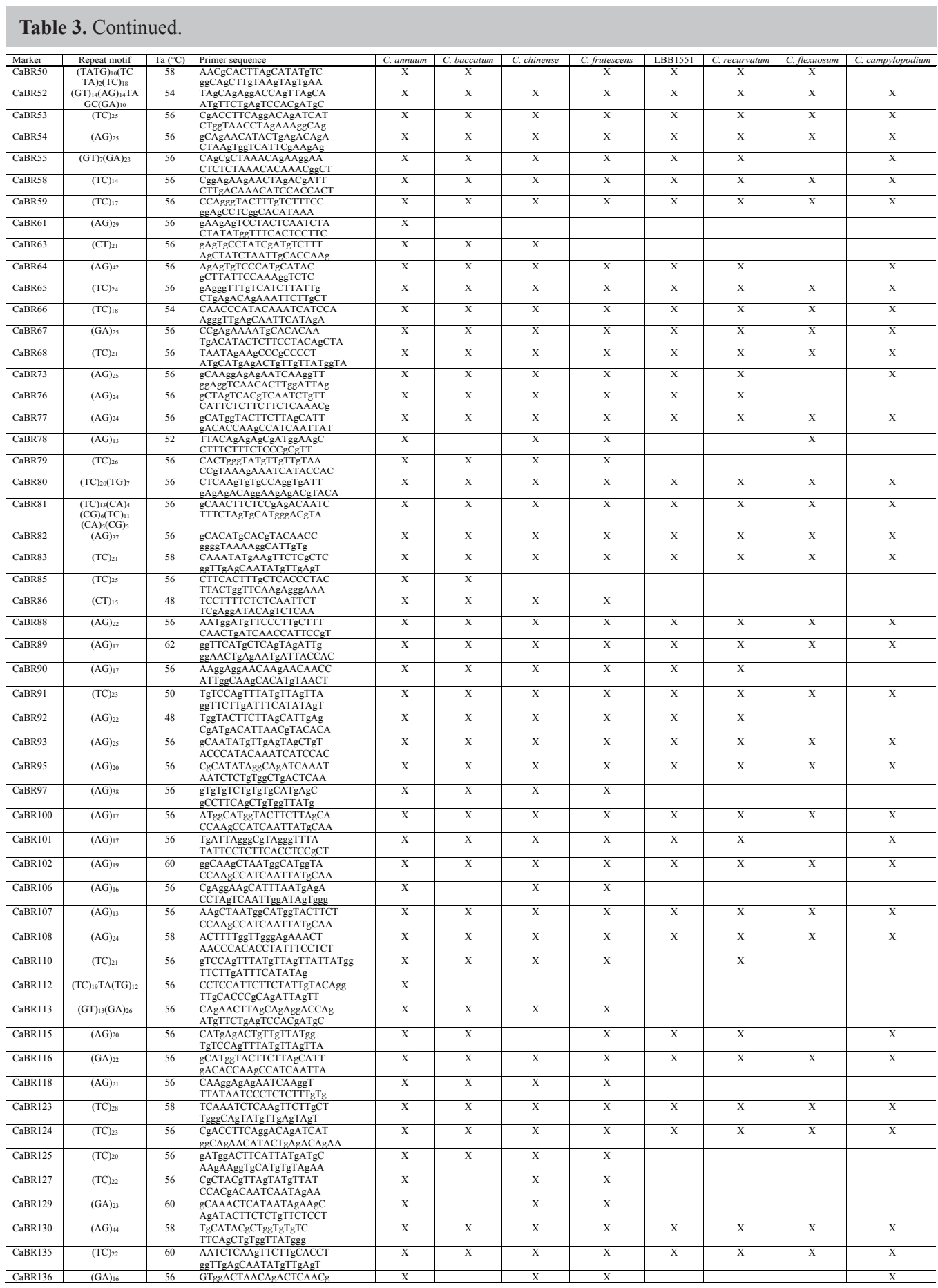

Continued on next page

Genetics and Molecular Research 15 (3): gmr.15038689 


\begin{tabular}{|c|c|c|c|c|c|c|c|c|c|c|c|}
\hline Marker & Repeat motif & $\mathrm{Ta}\left({ }^{\circ} \mathrm{C}\right)$ & \begin{tabular}{|l} 
Primer sequence \\
\end{tabular} & $\begin{array}{l}\text { C. annuиum } \\
\end{array}$ & C. baccatum & C. chinense & C. frutescens & LBB1551 & C. recurvatum & \begin{tabular}{|l|} 
C. flexuosum \\
\end{tabular} & C. campylopodium \\
\hline CaBRI36 & $(\mathrm{GA})_{16}$ & 56 & $\begin{array}{l}\text { GTggaCTAACAgACTCAACg } \\
\text { CTgATgATgCAgAACATgAT }\end{array}$ & $\mathrm{X}$ & & $\mathrm{x}$ & $\mathrm{x}$ & & & & $\mathrm{x}$ \\
\hline CaBR137 & (TC) 18 & 56 & \begin{tabular}{|l|} 
CgTCCTTTATTTCTTgCTT \\
AAAgAgAgAgATAgAAAggg \\
\end{tabular} & $\mathrm{X}$ & & $\mathrm{x}$ & $\mathrm{X}$ & & & & \\
\hline CaBR138 & $\left(\mathrm{TC}_{27}\right.$ & 56 & 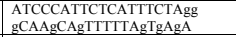 & $\mathrm{X}$ & & $\mathrm{x}$ & $\mathrm{X}$ & & & & \\
\hline CaBR139 & $(\mathrm{GA})_{7} \mathrm{C}_{4}(\mathrm{GA})_{19}$ & 56 & \begin{tabular}{|l|} 
gAAggATTACACAATATgTT \\
TTTCAAATCTgAAgTTCTCT
\end{tabular} & $\mathrm{x}$ & & $\mathrm{X}$ & & $\mathrm{x}$ & $\mathrm{x}$ & & \\
\hline CaBR140 & $(\mathrm{GA})_{12}$ & 58 & \begin{tabular}{|l|} 
gTgTgTCTgTgTgCATgAgC \\
TTCAgCTgTggTTATgggA
\end{tabular} & $\mathrm{X}$ & $\mathrm{X}$ & $\mathrm{X}$ & $\mathrm{X}$ & & & & \\
\hline CaBR141 & $(\mathrm{GA}) 13$ & 56 & $\begin{array}{l}\text { TTTCACCATgTTCAgATCC } \\
\text { CATCCATATATCgATCggCT }\end{array}$ & $\mathrm{X}$ & $\mathrm{X}$ & $\mathrm{X}$ & $\mathrm{X}$ & $\mathrm{X}$ & $\mathrm{X}$ & $\mathrm{x}$ & $\mathrm{x}$ \\
\hline CaBR142 & $(\mathrm{GA})_{12}$ & 58 & \begin{tabular}{|l|} 
gCCgATCgATATATggATgC \\
TTTgTgCCgCATCACTCC \\
\end{tabular} & $\mathrm{X}$ & $\mathrm{X}$ & $\mathrm{X}$ & $\mathrm{X}$ & $\mathrm{X}$ & $\mathrm{X}$ & & $\mathrm{x}$ \\
\hline CaBR143 & $\left(\mathrm{GA}_{21}\right.$ & 56 & \begin{tabular}{|l|} 
TATgCCCACCTCTAAAgAAA \\
AACCATCTgTTCATCTCCAT
\end{tabular} & $\mathrm{X}$ & $\mathrm{X}$ & $\mathrm{x}$ & $\mathrm{X}$ & $\mathrm{X}$ & $\mathrm{X}$ & $\mathrm{X}$ & \\
\hline CaBRI44 & $\left.(\mathrm{GT})_{S(G A}\right)_{21}$ & 56 & \begin{tabular}{|l|} 
TAgAAAACACAAAATgTCCCC \\
CCATTgACAAggACAATTCT
\end{tabular} & $\mathrm{x}$ & $\mathrm{X}$ & $\mathrm{x}$ & $\mathrm{x}$ & $\mathrm{x}$ & $\mathrm{x}$ & & $\mathrm{x}$ \\
\hline CaBR145 & $(\mathrm{CT})_{24}$ & 58 & \begin{tabular}{|l} 
CATTCTCTTCTTCTCAAACg \\
CACgTCAATCTgTTgTgAAA
\end{tabular} & $\mathrm{X}$ & $\mathrm{X}$ & $\mathrm{x}$ & $\mathrm{X}$ & $\mathrm{X}$ & $\mathrm{X}$ & $\mathrm{X}$ & $\mathrm{x}$ \\
\hline CaBR147 & $(\mathrm{AG})_{22}$ & 58 & \begin{tabular}{|l|} 
gAAgggTTTAgCAATATA \\
CTTAATTTCTCCTCTTC
\end{tabular} & $\mathrm{x}$ & $\mathrm{x}$ & $\mathrm{x}$ & $\mathrm{x}$ & $\mathrm{X}$ & $\mathrm{x}$ & & $\mathrm{x}$ \\
\hline CaBR149 & $(\mathrm{CT})_{4} \mathrm{~T}_{4}(\mathrm{CT})_{21}$ & 52 & $\begin{array}{l}\text { CAAACCATACAAATCATCTAAT } \\
\text { TTgTATTATTgAgAggA }\end{array}$ & $\mathrm{x}$ & $\mathrm{X}$ & $\mathrm{X}$ & $\mathrm{x}$ & $\mathrm{x}$ & $\mathrm{X}$ & $\mathrm{X}$ & $\mathrm{X}$ \\
\hline CaBR150 & $(\mathrm{AG}) / 4$ & 52 & \begin{tabular}{|l|} 
CTTCTTAgCATTgAgAgTTC \\
AATATCAgACACCAAgCC \\
\end{tabular} & $\mathrm{X}$ & $\mathrm{x}$ & $\mathrm{X}$ & $\mathrm{x}$ & $\mathrm{X}$ & $\mathrm{X}$ & & \\
\hline
\end{tabular}

\section{DISCUSSION}

The enrichment procedure used in this study proved to be effective and enabled the development of Capsicum DNA libraries enriched for microsatellite sequences. Across these libraries, approximately $50 \%$ of the sequenced clones contained short repeats. Brondani et al. (1998) used the same enrichment procedure and found similar results for eucalyptus. Of the 259 different microsatellite containing useful sequences for primer design, $83.8 \%$ were composed of perfect repeats, $9.3 \%$ of compound, and $6.9 \%$ of imperfect repeats. A high percentage of markers based on perfect repeats is desirable, especially for population studies, where allele calling of compound and imperfect repeat markers is complex and challenging.

The number of alleles per marker ranged from 2 to 11, with a mean of 6 alleles. This value is higher than that found in similar studies on self-pollinating plants (Hokanson et al., 1998) and in another study with peppers (Cheng et al., 2016), but similar to results obtained in Phaseolus vulgaris (Buso et al., 2006). The degree of polymorphism detected by the SSRs was measured using the PIC value. The PIC values obtained for the 45 Capsicum SSR markers among 48 genotypes averaged 0.60 . By comparison, gene diversity values and/or PIC values obtained for other autogamous species were found to be 0.43 for tomato (Panaud et al., 1996), 0.69 for rice (Bredemeijer et al., 1998), 0.63 for bean (Buso et al., 2006), 0.77 for Capsicum (Ibarra-Torres et al., 2015), and varying from 0.05 to 0.64 in Capsicum by Cheng et al. (2016). The information content of a marker can be determined by taking into account its ability to differentiate between individuals. The larger the number of alleles detected by a marker, the greater its potential utility appears to be. According to the criterion suggested by Botstein et al. (1980), a PIC value above 0.5 is considered high and indicates a high usefulness of the marker. Therefore, the set of Capsicum microsatellites reported here should be useful for germplasm characterization of both cultivated Capsicum as well as its wild relatives and might contribute to the understanding of the genetic relationships among Capsicum accessions, wild taxa, or both.

The characterization of the Capsicum germplasm, as reported for many other "orphan species", i.e., plant species for which we have little genetic and/or genomic knowledge, is usually limited by budget constraints. Thus, unequivocal identification of each accession is essential to avoid duplicate samples, which increases germplasm maintenance costs. The set 
of microsatellites described here, combined with appropriate allele-sizing algorithms and multiplex, semi-automated genotyping systems, could be used to characterize Capsicum germplasm collections. This would expedite the process of selecting representative germplasm sets for conservation, characterization, or breeding. In addition, because SSR markers are codominant, polymorphic, and stable, they should be very useful for genomic mapping and gene or QTL tagging, if a large number of SSRs is developed.

The ability to use the same SSR primers in different plant species depends on the extent to which the primer sites flanking SSRs are conserved between related taxa. The cross-species transferability of SSR markers in Capsicum was found to be high, with high percentages of loci producing amplicons in all tested species. This indicates that a high level of sequence conservation exists within the primer regions of these Capsicum species. The high transferability rate of the SSR loci indicates that these markers are powerful tools for synteny analysis in Capsicum. Furthermore, our study identified novel candidate SSR markers for C. flexuosum, C. recurvatum, and C. campylopodium, which had not been investigated in previous Capsicum SSR development studies.

The evolutionary conservation of these loci allows for successful cross-species amplification using the same primers and amplification conditions. This facilitates their potential use in phylogeny studies, investigations into levels of genetic variation, and other population parameters such as gene flow in the different species of the Capsicum genus. Recently, Ibarra-Torres et al. (2015) used SSR markers originally developed for C. annuиm to analyze $C$. pubescens genotypes showing high genetic homology among both species. Ince et al. (2010) also reported that microsatellite primer pairs amplified genomic targets of $C$. annuum, C. baccatum, C. chacoense, C. chinense, C. frutescens, and C. pubescens, also indicating species transferability within Capsicum. In beans, Yu et al. (2000) identified SSR primers from Vigna aconitifolia, a distantly related species, which amplified Phaseolus vulgaris DNA. Ashkenazi et al. (2001) identified SSR markers obtained from tomato capable of producing PCR products from the potato genome.

In conclusion, we report the development of a new set of SSR markers for Capsicum that is immediately available for use by the scientific community. The possible applications of these markers range from cultivar identification, analysis of genetic diversity to trait mapping and marker-assisted selection.

\section{Conflicts of interest}

The authors declare no conflict of interest.

\section{ACKNOWLEDGMENTS}

The authors thank the Brazilian Agricultural Research Corporation (EMBRAPA) for financial support.

\section{REFERENCES}

Ashkenazi V, Chani E, Lavi U, Levy D, et al. (2001). Development of microsatellite markers in potato and their use in phylogenetic and fingerprinting analyses. Genome 44: 50-62. http://dx.doi.org/10.1139/gen-44-1-50

Bassam BJ, Caetano-Anollés G and Gresshoff PM (1991). Fast and sensitive silver staining of DNA in polyacrylamide

Genetics and Molecular Research 15 (3): gmr.15038689 
gels. Anal. Biochem. 196: 80-83. http://dx.doi.org/10.1016/0003-2697(91)90120-I

Botstein D, White RL, Skolnick M and Davis RW (1980). Construction of a genetic linkage map in man using restriction fragment length polymorphisms. Am. J. Hum. Genet. 32: 314-331.

Bradeen JM and Simon PW (1998). Conversion of an AFLP fragment linked to the carrot $Y_{2}$ locus to a simple, codominant, PCR-based marker form. Theor. Appl. Genet. 97: 960-967. http://dx.doi.org/10.1007/s001220050977

Bredemeijer GMM, Arens P, Wouters P, Visser D, et al. (1998). The use of semi-automated fluorescent microsatellite analysis for tomato cultivar identification. Theor. Appl. Genet. 97: 584-590. http://dx.doi.org/10.1007/s001220050934

Brondani RPV, Brondani C, Tarchini R and Grattapaglia D (1998). Development, characterization and mapping of microsatellite markers in Eucalyptus grandis and E. urophylla. Theor. Appl. Genet. 97: 816-827. http://dx.doi. org $/ 10.1007 / \mathrm{s} 001220050961$

Buso GSC, Amaral ZPS, Brondani RPV and Ferreira ME (2006). Microsatellite markers for the common bean Phaseolus vulgaris. Mol. Ecol. Notes 6: 252-254. http://dx.doi.org/10.1111/j.1471-8286.2006.01210.x

Cheng J, Zhao Z, Li B, Qin C, et al. (2016). A comprehensive characterization of simple sequence repeats in pepper genomes provides valuable resources for marker development in Capsicum. Sci. Rep. 6: 18919. http://dx.doi. org/10.1038/srep18919

Dias GB, Gomes VM, Moraes TM, Zottich UP, et al. (2013). Characterization of Capsicum species using anatomical and molecular data. Genet. Mol. Res. 12: 6488-6501. http://dx.doi.org/10.4238/2013.February.28.29

Doyle JJ and Doyle JL (1987). Isolation of plant DNA from fresh tissue. Focus 12: 13-15.

Hokanson SC, Szewc-McFadden AK, Lamboy WF and McFerson JR (1998). Microsatellite (SSR) markers reveal genetic identities, genetic diversity and relationships in a Malus x domestica borkh. core subset collection. Theor. Appl. Genet. 97: 671-683. http://dx.doi.org/10.1007/s001220050943

Ibarra-Torres P, Valadez-Moctezuma E, Pérez-Grajales M, Rodríguez-Campos J, et al. (2015). Inter- and intraspecific differentiation of Capsicum annuum and Capsicum pubescens using ISSR and SSR markers. Sci. Hortic. 181: 137146. http://dx.doi.org/10.1016/j.scienta.2014.10.054

Ince AG, Karaca M and Onus AN (2010). Polymorphic microsatellite markers transferable across Capsicum species. Plant Mol. Biol. Report. 28: 285-291. http://dx.doi.org/10.1007/s11105-009-0151-y

Kong Q, Zhang G, Chen W, Zhang Z, et al. (2012). Identification and development of polymorphic EST-SSR markers by sequence alignment in pepper, Capsicum annuum (Solanaceae). Am. J. Bot. 99: e59-e61. http://dx.doi.org/10.3732/ajb.1100347

Lee JM, Nahm SH, Kim YM and Kim BD (2004). Characterization and molecular genetic mapping of microsatellite loci in pepper. Theor. Appl. Genet. 108: 619-627. http://dx.doi.org/10.1007/s00122-003-1467-x

McLeod MJ, Eshbaugh HW and Guttman SI (1979). A preliminary biochemical systematic study of the genus Capsicum - Solanaceae. In: The biology and taxonomy of the Solanaceae (Hawkes JG, Lester RN and Skelding AD, eds.). Academic Press, New York, 701-714.

Minamiyama Y, Tsuro M and Hirai M (2006). An SSR-based linkage map of Capsicum annuum. Mol. Breed. 18: 157-169. http://dx.doi.org/10.1007/s11032-006-9024-3

Nagy I, Stágel A, Sasvári Z, Röder M, et al. (2007). Development, characterization, and transferability to other Solanaceae of microsatellite markers in pepper (Capsicum annuum L.). Genome 50: 668-688. http://dx.doi.org/10.1139/G07-047

Nicolaï M, Pisani C, Bouchet JP, Vuylsteke M, et al. (2012). Discovery of a large set of SNP and SSR genetic markers by high-throughput sequencing of pepper (Capsicum annuum). Genet. Mol. Res. 11: 2295-2300. http://dx.doi. org/10.4238/2012.August.13.3

Panaud O, Chen X and McCouch SR (1996). Development of microsatellite markers and characterization of simple sequence length polymorphism (SSLP) in rice (Oryza sativa L.). Mol. Gen. Genet. 252: 597-607.

Paran I, Aftergoot E and Shifriss C (1998). Variation in Capsicum annuum revealed by RAPD and AFLP markers. Euphytica 99: 167-173. http://dx.doi.org/10.1023/A:1018301215945

Portis E, Nagy I, Sasvári Z, Stágel A, et al. (2007). The design of Capsicum spp. SSR assays via analysis of in silico DNA sequence, and their potential utility for genetic mapping. Plant Sci. 172: 640-648. http://dx.doi.org/10.1016/j. plantsci.2006.11.016

Prince JP, Loaiza-Figueroa F and Tanksley SD (1992). Restriction fragment length polymorphism and genetic distance among Mexican accessions of Capsicum. Genome 35: 726-732. http://dx.doi.org/10.1139/g92-112

Prince JP, Pochard E and Tanksley SD (1993). Construction of a molecular linkage map of pepper and a comparison of synteny with tomato. Genome 36: 404-417. http://dx.doi.org/10.1139/g93-056

Sambrook J, Fritsch EF and Maniatis T (1989). Molecular cloning: a laboratory manual. 2nd edn. Cold Spring Harbor Laboratory Press, New York.

Sanwen H, Baoxi Z, Milbourne D, Cardle L, et al. (2000). Development of pepper SSR markers from sequence databases. Euphytica 117: 163-167. http://dx.doi.org/10.1023/A:1004059722512

Genetics and Molecular Research 15 (3): gmr.15038689 
Sugita T, Semi Y, Sawada H, Utoyama Y, et al. (2013). Development of simple sequence repeat markers and construction of a high-density linkage map of Capsicum annuum. Mol. Breed. 31: 909-920. http://dx.doi.org/10.1007/s11032013-9844-x

Untergasser A, Cutcutache I, Koressaar T, Ye J, et al. (2012). Primer3 - new capabilities and interfaces. Nucleic Acids Res. 40: e115. http://dx.doi.org/10.1093/nar/gks596

Yi G, Lee JM, Lee S, Choi D, et al. (2006). Exploitation of pepper EST-SSRs and an SSR-based linkage map. Theor. Appl. Genet. 114: 113-130. http://dx.doi.org/10.1007/s00122-006-0415-y

Yu K, Park SJ, Poysa V and Gepts P (2000). Integration of simple sequence repeat (SSR) markers into a molecular linkage map of common bean (Phaseolus vulgaris L.). J. Hered. 91: 429-434. http://dx.doi.org/10.1093/jhered/91.6.429

Genetics and Molecular Research 15 (3): gmr.15038689 